\title{
Usalpharma: A Software Architecture to Support Learning in Virtual Worlds
}

\author{
Juan Cruz-Benito, Cristina Maderuelo, Francisco J. García-Peñalvo, Roberto Therón, Jonás Samuel \\ Pérez-Blanco, Hinojal Zazo, Ana Martín-Suárez
}

\begin{abstract}
This paper explains how a software architecture was planned, designed and implemented to help in the support of eLearning activities within a virtual laboratory inside the Second Life Virtual World. It delves into the problems related to this kind of systems and architectures and detects the possible benefits they could provide to eLearning processes. The paper shows how the software engineering principles and procedures are applied in order to solve problems like data gathering from Virtual Worlds, data analytics of information related to interaction between a user and a 3D environment, and how they can be applied to enhance the students' learning process and teachers' assessment of this learning process. This paper also shows the software product resulting of this engineering process, as well as the outcomes from the application of the solution in a real context during two academic years involving postgraduate students and subjects of Pharmacy and Quality Assurance. To end this paper, the authors explain some considerations and knowledge retrieved after this experience, focusing on the utility from the learning point of view, innovation and possible future work that could be done to improve the solution implemented.
\end{abstract}

Index Terms-Software Architectures, Virtual Worlds, Technology-Enhanced Learning, Usage analytics

\section{INTRODUCTION}

$\mathrm{E}$ NGINEERING as a discipline is, according to [1], "the

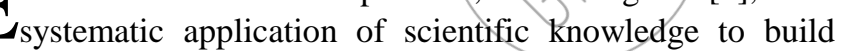
solutions, in an effective and economically viable way, for practical problems in the service of humanity"; moreover, in the case of software engineering, it is sometimes defined as "the application of a systematic, disciplined and quantifiable development, operation and maintenance of software; i.e. the application of engineering to software " [2].

Engineering is a sort of Swiss Army knife that gives those who use it (engineers) the necessary tools to solve problems by

Juan Cruz-Benito and Francisco J. García-Peñalvo are with the GRIAL Research Group, Computer Science Department, Research Institute for Educational Sciences, University of Salamanca, Salamanca 37008, Spain (emails: juancb@usal.es y fgarcia@usal.es).

Roberto Theron is with the GRIAL Research Group, Computer Science Department, and Vis-USAL Research Group, University of Salamanca, Salamanca 37008, Spain (email: theron@usal.es)

Cristina Maderuelo, Jonás Samuel Pérez-Blanco, Hinojal Zazo and Ana Martín-Suárez are with the Department of Pharmacy and Pharmaceutical Technology, University of Salamanca, Salamanca 37008, Spain (e-mails: cmaderuelo@usal.es, jsperez@usal.es, hinojal@usal.es y amasu@usal.es) varying these tools depending on the area where engineers apply their knowledge or the nature and complexity of the problem to be solved. Still furthermore, the application of this Swiss Army Knife is not banned to non-engineers: Engineers use it and know better than anyone how to use it, but its use can benefit the whole society. Even more specifically, it can benefit communities of individual users or work teams regardless of their status, area of knowledge and experience, to solve different kinds of problems.

This article, entitled "Usalpharma: A Software Architecture to Support Learning in Virtual Worlds," presents how engineering has been used to solve a real problem in an educational context. It describes how a multidisciplinary team composed of profiles in the world of Computer Engineering and the area of Health Sciences (specifically the area of Pharmacy) have been able to solve a complex problem, a priori, such as monitoring student activity within the context of a Virtual World to assist in the evaluation of educational activities worldwide, through the application of technical and engineering tools (in this case software engineering) [3]. Related to the application of software engineering in a multidisciplinary context and in educational settings still in development, it should be noted that this project is neither unique nor pioneer in this type of collaboration and expansion of the application spectrum of technology solutions to service education, but it brings its vision and specific solutions within the set of the current generation of education systems enhanced by technology and current trends in technology to support processes of acquisition of knowledge and skills in heterogeneous learning environments.

To illustrate this particular case, this article is divided into the following sections (in addition to this first introductory section): the second section, Usalpharma: Educational Environment in a Virtual World, accurately describes the context of the problem to be solved, introducing the reader to the virtual environment where the teaching of students in the knowledge area of pharmacy is developed, helping to understand the rest of the article. The third section, The Problem: Proposal, Objectives and Requirements, poses the challenges faced by the multidisciplinary team, as well as the objectives and requirements that the solution must meet. The fourth section, Solution and Product Developed, specifies the theoretical solution that was proposed after applying an approach of software engineering to the problem, and the 
translation of this theoretical solution in a tangible software product and which can be used in a real context as presented. The fifth section, Exploitation and Results, presents the experience gained after applying this software solution for two academic years in the activity carried out within the context of the virtual world Second Life, as well as a number of indicators of use and usefulness of that solution. The sixth section, Discussion, comments on the main aspects of the work that has been carried out, trying to enlighten the reader about the satisfactory and unsatisfactory aspects of the proposed solution and the experiment carried out, always from a standpoint of reflection and awareness of the results and their significance. Finally, the seventh section, Conclusions and Future Work, summarizes the results in general and a series of lines that open future work on this project.

\section{USALPHARMA: EDUCATIONAL ENVIRONMENT IN A VIRTUAL WORLD}

Usalpharma Lab is a virtual laboratory built by the teaching innovation group Usalpharma and the Department of Pharmacy and Pharmaceutical Technology of the University of Salamanca, which is within the Virtual World Second Life [4]. The laboratory simulates the facilities, equipment, documentation and tools afforded by a real laboratory of the pharmaceutical industry that complies with GLP (Good Laboratory Practices) regulations and has been used in teaching since 2011 to train graduate students in subjects related to Quality in the Pharmaceutical Industry [5, 6]. The reason for building a virtual scenario of this type of laboratory is the economic cost and physical space that must be used, non-asumible for a university or any other institution that would not get any direct economic profit from it.

The training that students receive/in this virtual scenario is based on learning methodologies, standards and audit processes in laboratories of the pharmaceutical industry in a practical way (and not merely theoreticalty, as had been usual), through interaction with a 3D scenario that replicates a laboratory that represents a real case. To do this, students act within virtual facilities as true external auditors who are responsible for assessing compliance with the laboratory of these GLP measures, generating a report outlining compliance (or not) of the rules at the end of practical sessions, flaws identified in it (there are always a number of deficiencies brought intentionally by teachers), critical nature, etc. This type of training has been included since 2011 as part of the subject Quality Assurance in the Laboratory Analysis in the Pharmaceutical Industry within the Master in Drugs Evaluation and Development of the University of Salamanca (Spain).

\section{The Problem: Proposal, OBJectives AND REQUIREMENTS}

This section presents the problem proposed by teachers and technical personnel responsible for the Usalpharma Lab and the specific objectives to be met by the solution, as well as the software requirements developed to solve the problem.

\section{A. The proposal of the problem}

Between 2011 and 2013 this scenario was used in teaching under the direct supervision of teachers of the course and staff involved in the development of 3D environments. That is, practice work used to be developed in the laboratory (in a single session and call for all students), was guided by a teacher and was supervised by the rest of the team, so that if a student had any questions or appreciation of the 3D scenario, content resources, possible variations or disagreements regarding present rules or any other aspect they could immediately make a query, getting instant feedback and sometimes very specific help. This stage, as far as results are concerned, was very positive for the initiative, students got good grades in their practice and showed satisfaction with the methodology and resources used in this virtual learning [7].

After this first stage in the implementation of this type of system to support teaching, the teaching innovation team wanted to go a step further, through implementing a system that would replace the teacher's guide during the students' practical sessions, controlling whether students were doing or not the practice within the virtual world, as well as to include a number of requirements on the feedback that students receive when participating in their practical work. That is, a system that allows taking advantage of the potential of virtual worlds in terms of the ability of autonomous learning, the possibility of not depending on the time and physical space (time, physical location of students) to leverage resources provided by virtual learning environments as well as presenting better immersive experiences to students within the $3 \mathrm{D}$ environment [8-12].

\section{B. Specific objectives}

Then, the proposed objectives for this system (both objectives proposed in the first version of the system, as well as those proposed for successive versions) are as follows:

- The system should be able to monitorize what students do in the virtual laboratory, discriminating who clicks on any object, when they do, and what object or part of the particular installation is used.

- This system should allow the teacher to know the actions carried out by each student, showing him or her some kind of report associated with each student or user registered by the system. These actions can be consulted unformatted (or in minimum formats such as lists or tables) and be filtered using temporal parameters (date range), by object type, etc.

- The system should control if the student is carrying out the necessary checks on those sensitive elements to breach the rules, especially those that teachers marked incorrectly on the virtual stage. The result of these checks should be included in the report on the activity of each student.

- The system should provide general reports on the activity of a group of users at any given time, not only individually for each registered user. 
- The system must be able to transmit real-time feedback to students on their progress in their practical work.

\section{Software requirements}

Among the determined requirements for this system the following were mainly specified:

- The system should store for each action performed in the virtual world (click on objects) the name of the user performing the action, the name or descriptor of the object that receives the action, the specific time when that has been done (timestamp) as well as the specific location where the action was performed.

- The system should show teachers a report of students' actions regardless of device and operating system from which the report was accessed.

- The system should provide in-world feedback to students, that is, it must have the necessary tools not only to extract information from Virtual World, but also to provide input.

- The system must efficiently store the required data. The data layer should allow varying the model and the data types that support, in anticipation of possible changes suffered by the system in the future, or additional features that should be contemplated.

- The system must be prepared to incorporate new sources of data (student grades, personal data, integration with other platforms, etc.) in the possible future.

Regarding other possible requirements to be specified (functional, non-functional or others for information) as the technology that must be employed, modeling specific data, or aspects of extensibility and modularity of the system, all the team members agreed to request these aspects to the engineers, so they evaluated what might be the best option in each case.

\section{Solution ANd PRodúct DEVEloped}

In this section are detailed three fundamental parts of the search process of a proper solution to the proposed problem: previous considerations, the theoretical solution proposed and the final developed product that is currently on production.

\section{A. Previous considerations to the design of the solution}

Before designing and developing a viable and optimal solution for this problem, researchers had to take into account a number of specific preliminary considerations of the problem to be solved, among which are highlighted:

- The problems posed by the interconnection of a private and closed system such as Second Life with any external platform. Not only does it present problems regarding integration, there are no public resources, logs, APIs or other tools to integrate with other systems [13, 14] beyond basic information on number of users per day, etc. [15]. It presents additional problems because of the rigid characteristics regarding the ownership of the 3D objects, which hinders the usage for the described purpose. Even more, Second Life is provided "as is", which prevents any claims about failures, changes, etc. and requires users to modify the system behaviour [16].

- The system that would be proposed as a solution must be able to coordinate the response to the whole problem, from data collection to the presentation of the same on any device or within the Virtual World. This implied drawing on wellknown open technologies, standards that have an almost complete presence in every possible system. These standards must be present in the technological solution as well as communication protocols, data collection, etc. [17, 18].

- The technology that supports the management of processes, such as layer data management, must be able to withstand peaks of activity (many students interacting simultaneously in the 3D laboratory), possible massive load at certain time intervals (while carrying out the practical work) and concurrency in the interaction with the resources that provides performance without excessive erosion.

\section{B. Theoretical solution proposed}

Since 2013 the authors have been working to address the problem in a satisfactory manner, and in early 2014 a viable solution was finally proposed [14] (at least from a theoretical point of view) that provided an adequate framework and a proof of concept for the final development of a product. This solution could remedy the problem regarding integration of a system that monitorizes, analyzes, reports and helps the evaluation of teaching activities within the virtual laboratory Usalpharma Lab.

This solution is based on an architecture that could be deployed in a cloud environment so that the architecture, based on the typical client-server schemes, consisted of a number of layers (data collection, data persistence of analysis, presentation, etc.) connected to each other in the same way (client-server depending on which requires the services of other interactions), enabling the deployment of each layer even in different clouds (with the potential to scale only those resources required, apply different technologies to each layer, etc.) due to the fact that the layers simply interact among them using services (Figure 1). 


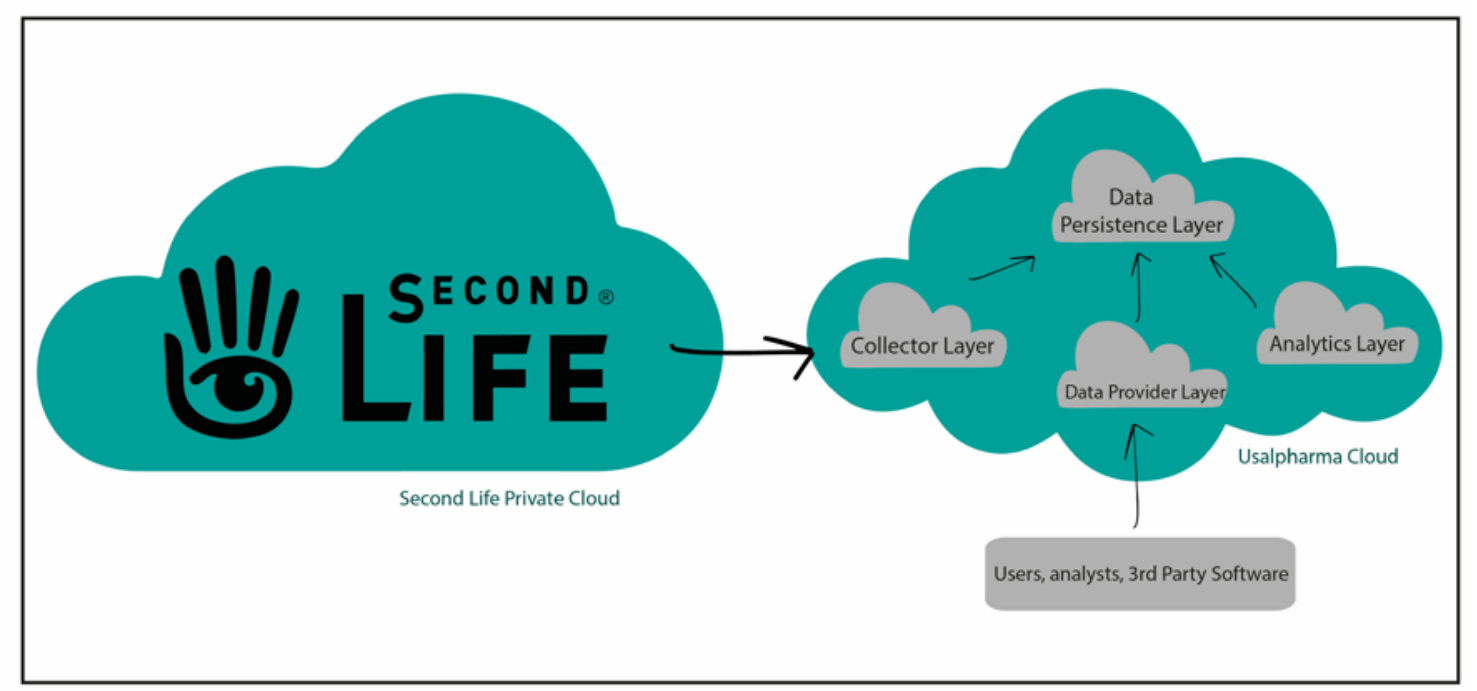

Fig. 1. Simplified representation of the architecture of the proposed solution [14]

Regarding the standards and technologies proposed in this theoretical solution, the following can be outlined:

- Use of a (simplified) variation of the semantic standard RDF and its triples [19, 20] to transmit structured and rich information about the interaction in the Virtual World to the data collection layer.

- Use of the HTTP protocol [21] for the transmission of messages between the Virtual World and the layers of data collection and provision (in both directions, the Virtual World to the system, and the system to the Virtual World), specifically using the part of the protocol concerning operations, requests, status codes, and headers.

- Intensive use of web technologies to return information to users (teachers and students) regardless of device, operating system or software (such as Second Life viewer).

- Using NoSQL documents-oriented databases (MongoDB [22] specifically) that allow modification of data architecture transparently and without penalty arising from the persistence of the layer architecture (only possible penalties in the logical layer derived from changes in its own data models [23]), and maintaining good performance in information processing, and high scalability in production environments. [24].

- Use of server technologies that have demonstrated their capacity for data processing HTTP requests and adequate performance under heavy demand. In this case, the technology proposed was the web framework Django [25], which develops robust solutions using Python and has many libraries of its own (and others of the language) to facilitate the development of stable agile projects.

This solution provides a flow of activity as proposed in the activity diagram shown in Figure 2, which is an updated version of the software's architecture of the version currently implemented. In this diagram, the interaction can be seen between the system and the actors (stakeholders) involved in it, and serves as a summary of the workflows of the whole process.

\section{Product developed}

In this third part of the section are detailed the various major components of the product developed from the theoretical proposal for solving the problem. This product consists of three main parts, architecture and server itself, as well as Web clients (for teachers) and client embedded in Second Life (for students):

Software Architecture: As previously discussed, it is implemented using the web framework Django, and consists of a series of applications that are responsible for collecting the HTTP requests that launches the Second Life client for recording data interaction, cleaning and processing such information, contacting the persistence layer data and database MongoDB data, serving requests such as "display information" depending on the context / client from which they are requested (the same information is not served when the user launches requests from the web application or from the Virtual World), calculating data associated with user interaction: measures of the time spent by each student to complete their practical work [26], calculating the most relevant key points achieved [27], reporting for each user or group of users, etc. These applications and the multiple layers composed architecture are responsible for maintaining the logic of the whole system as well as the bulk of the functionality.

Web Client: From the Web client, teachers can consult all data concerning students and their work progress. Among the metrics that can be known should be available the number of collected interactions, time spent by students performing, the achievement by the students of the inspection of the various elements (review of safety measures laboratory, review of equipment to be audited, documentation, etc.) as well as 
comprehensive reports for each class of students, so that a teacher can know in a detailed way the performance of each student, or acquire an overview of the group of students in their practicals. This web client is developed to be used from any device (PC, tablets, smartphones, etc.). Figure 3 presents some data visualization of various metrics that teachers can know about student performance in Usalpharma Lab.

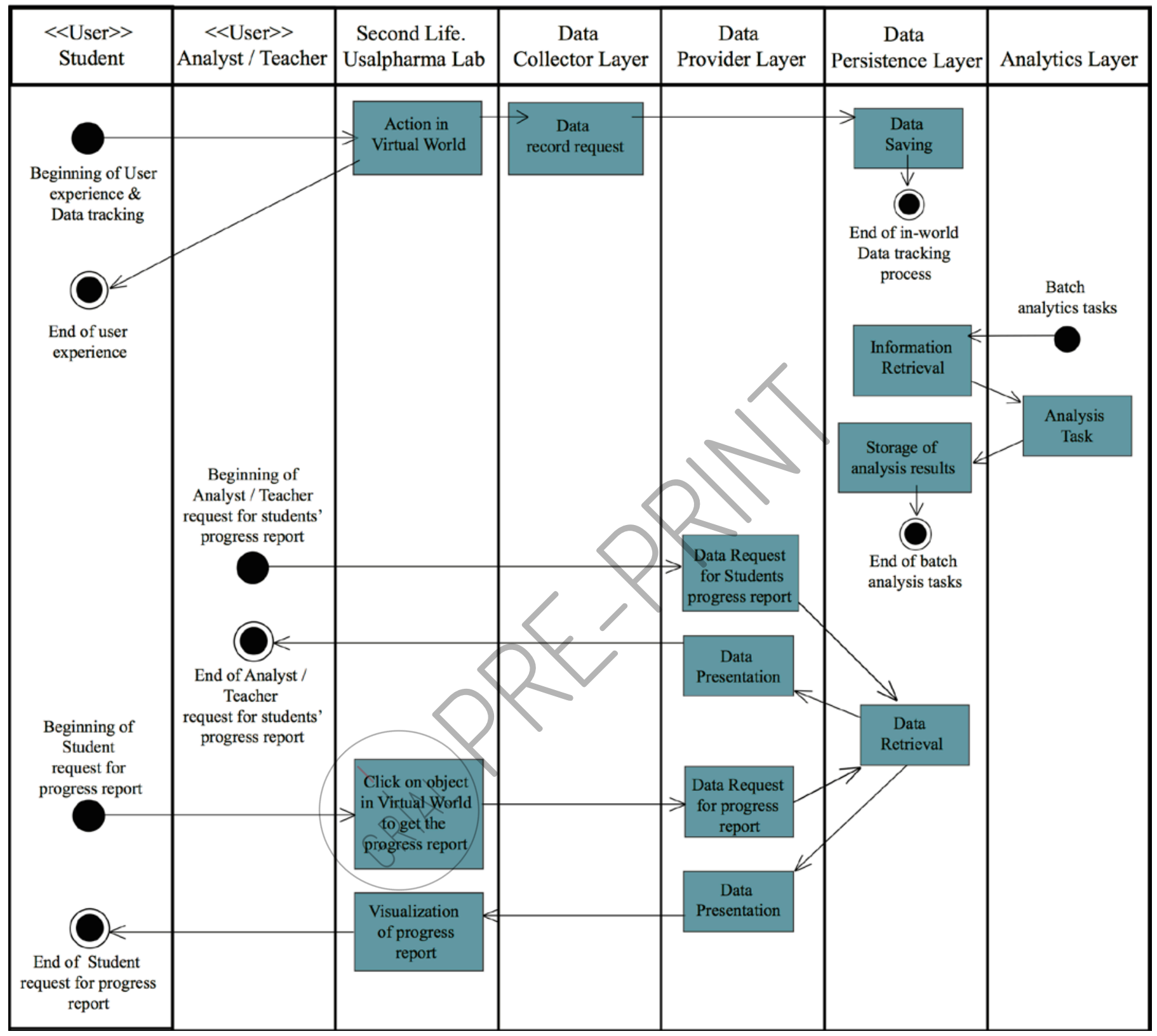

Fig. 2. Activity diagram among users, Virtual World and system. Updated version of the proposed in [14] 


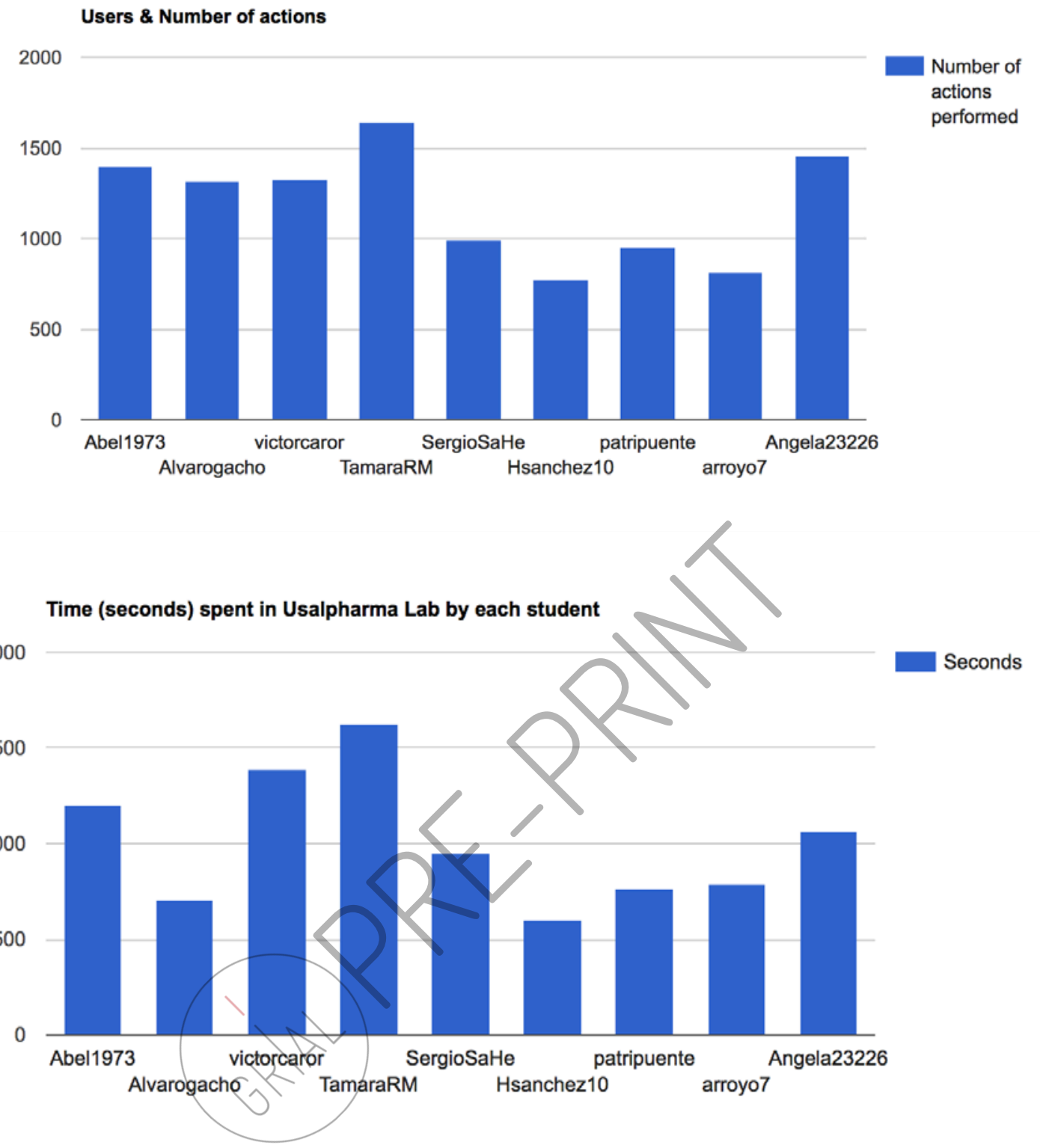

Users \& Checkpoints achieved

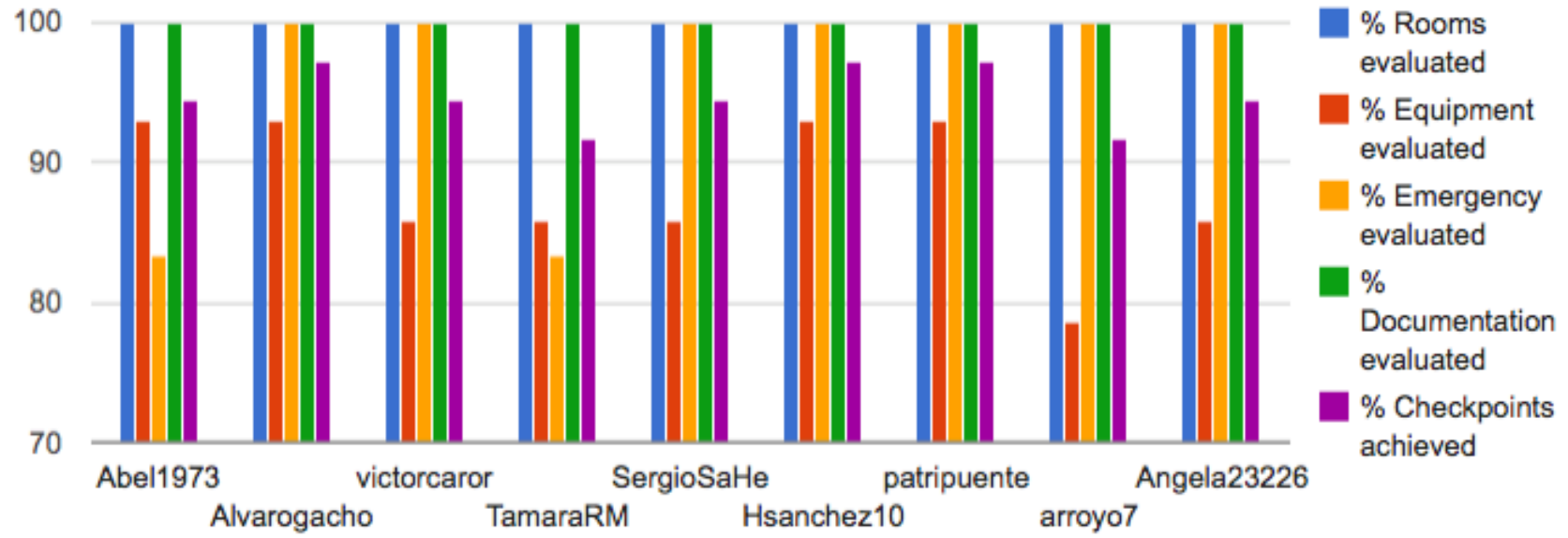

Fig. 3. Some data visualization presented in the Web client. They show different metrics related to student activity in the 2015 practice 
Second Life client: This client is somewhat different from the web client because it can be used by any avatar (user in Virtual World) that is in the laboratory. The client is used through interaction (click) on an object in the laboratory, so that the student will see a dialog showing in real time the percentage of critical points assessed, both in the day when a query is made and, in general, making suggestions on what the student should re-audit. In the first year of practice the system showed only feedback about the particular day of the request, without giving an overview of the development of the practical on different days given to audit. Figure 4 shows this type of activity report.

At the same time, the Second Life client has an even more essential role, and that is to send data to the system, so that it registers every evidence of interaction between the user and the virtual laboratory (data entry to the platform). To do this, all 3D objects to be audited by students in the virtual laboratory have an associated script that throws a HTTP request to the application, which is responsible for collecting information about user interaction to record the action that is occurring at the moment. This request is carried out silently and transparently for the user; the object itself is responsible for collecting the data on the date and the exact moment of interaction (timestamp), with what the user has interacted, where in the virtual lab it is, etc., so that the user is not aware of being transmitting that information to the system, although all students are previously notified of the monitoring carried out in the laboratory.

\section{Evolution and improvement of the proposal}

As mentioned, in 2014 a first formal proposal was made and published, explaining how to address the problem [14]. In that first proposal a system was presented that would allow the collection of learning evidences and that could offer teachers some level of information about what students performed within the virtual laboratory environment. It also focused on the problems that such proposal must face from the point of view of software engineering to obtain information from the virtual world and take proper advantage of it as an evidence of learning by defining and using RDF simplified schemes, making data collection through structures and information architectures flexible enough to vary the type of information retrieved, and allowing the interoperability of the data schema to other systems (new developments or third-party systems) in a simple and transparent way.

After this initial proposal, the project came under development and, based on the first results and observed possibilities, the authors saw the opportunity to expand the system functionality, including a few months after the first enforcement and feedback mechanisms within the 3D laboratory [27] and opening the way for learning personalization and adaptivity of learning (referred as part of future work, as will be discussed at the end of the paper).

Once implemented these features and having a sufficiently functional system, it was tested with real users (years 20132014 and 2014-2015). These tests were helpful to verify the actual usefulness of the system and its operation. Likewise, once the system had been put to production, the authors made improvements in the reporting of users (students in the Virtual World, teachers using the Web client, etc.).

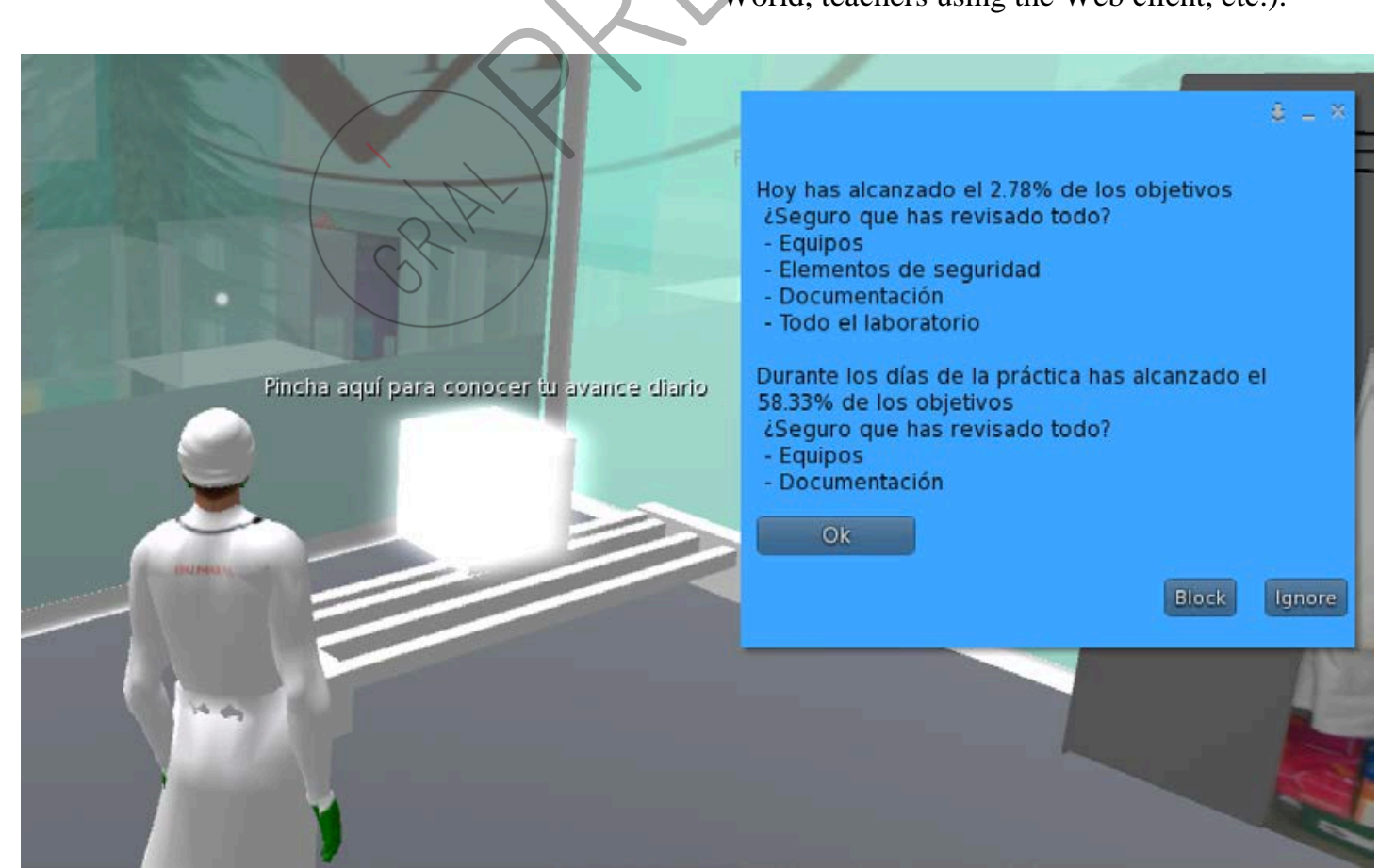

Fig. 4. Real-time information that students can get in Second Life about the audit performed. In the Spanish text that appears in the figure, the system informs the student about the performance (\% of the goals in the day and in all the days of the practice) and suggests the different elements where the student has not completed the audit. 


\section{EXPLOITATION AND RESULTS}

The solution developed has been in production since February 2014, monitoring continuously since that time (excluding time spent on upgrading the system to the latest versions) any relevant activity that has occurred in the laboratory. Specifically, the system has already monitorized two real practicals with students, one in the course 2013-2014 (between 6 and 24 March 2014) and another in 2014-2015 (between 5 and 12 March 2015) of the aforementioned subject Quality Assurance in the Laboratory Analysis in the Pharmaceutical Industry within the Master in Drugs Evaluation and Development of the University of Salamanca (Spain). The system has also monitorized the interaction that other users have had during the time elapsed between such practicals. These have involved 18 students (9 in the 2013-2014 year, 9 in the 2014-2015 year) that were enrolled in the subject.

In such practice sessions, these tools presented here have been taken into account for the assessment of the subject, because teachers have decided to give a part of the evaluation of practice to the data provided by the system. Specifically, 25 percent of the practice mark directly depends on the percentage of critical points assessed by the student in the laboratory (\% checkpoints achieved in Figure 3). The other $75 \%$ of the practice mark corresponds to the audit report presented by students at the end of their practice sessions, and where they really show their knowledge about the GLP regulations and the elements of the laboratory that meet them or not. Other data taken into account when assessing the practicals are: a) if the student has carried out work on the agreed dates, b) if the student has made more than 1 hour of audit (in one or more sessions), c) if they have reached more than $12 \%$ of checkpoints revised. The teaching staff sets these three elements as the minimum requirements to show that students have actually audited and evaluated the laboratory.

Among the different results that can be shown to illustrate the operation of the entire system, it is possible to distinguish between two vectors of data to help test whether the system works, and to indicate if the system may be accepted for its actual use in teaching:

- Data concerning the validity of the system to help students practice and somehow replace a personal support teacher in the virtual lab.

- Data about measurements that show the usefulness of a system of collection, sorting and processing of data in a case like this.

In the first case (validity of the system to help students practice and somehow replace personal support teachers in the virtual lab), in order to judge the results it is possible to compare the average marks obtained by students in the different years that the virtual laboratory has been used for practicals (years with direct support of the teachers as well as years with the implementation of this architecture and software tools). Table 1 shows a summary of the marks:

$$
\text { TABLE I }
$$

STUDENTS AND THE AVERAGE MARKS CORRESPONDING TO THE COURSES WHEN PRACTICALS WERE REALIZED

\begin{tabular}{cccc}
\hline \hline Course & $\begin{array}{c}\text { Number of } \\
\text { students }\end{array}$ & $\begin{array}{c}\text { Average mark } \\
\text { obtained }(0 \text { to } \\
10)\end{array}$ & $\begin{array}{c}\text { Standard } \\
\text { deviation }\end{array}$ \\
\hline $2011-2012$ & 14 & 7.28 & 1.38 \\
$2012-2013$ & 16 & 7.5 & 1.27 \\
$2013-2014$ & 9 & 7.81 & 0.7 \\
$2014-2015$ & 9 & 8.54 & 0.56
\end{tabular}

Based on these results, it is possible to see how students have increased their average mark (and decreasing the standard deviation) in each year, showing no significant changes in the trend between the different years -namely, the disparities observed between those years where the practice was conducted as a single session with teachers' guidance and the other years where the practice was aided using the complementary technological tools developed within the scope of this project. As will be discussed in the discussion section, these data do not have to convey that the use of this architecture and the tools provided improve the results of practical sessions (this may be due to many factors, such as the decline in number of students, the possible prior habituation of students to similar tools or 3D contexts, etc.), but it illustrates how a trend of improving results is maintained even though the method of help, support and evaluation of the practicals varies.

As for the second information vector, about the usefulness of this type of system for monitoring, reporting results and aiding in the evaluation and development of practical work, a series of metrics can be displayed collected by the platform that illustrate the reader about the volume of information that the system has collected in these two academic years (20132014 and 2014-2015, specificly between 18 February 2014 and 20 March 2015), saving teachers from:

- 24276 interaction evidences (clicks in the laboratory).

- 249 sessions by 47 different users (teachers, students and visitors to the facilities).

- 354269 seconds of interaction registered (total time of use of the system by the users, measured in seconds). It is more than 98 hours of interactions retrieved, stored and analyzed for use by teachers. Of this usage time, 137454 seconds (over 38 hours) of use of the laboratory correspond with students who carried out their practical work in the year 2013-2014 and 136581 seconds (almost 38 hours, again) of usage correspond to the time students who participated in the year 2014-2015 (in total, these students have registered almost $78 \%$ of total usage time in the laboratory).

These data are only a sample of what the Usalpharma architecture has contributed to the development of practice and the enormous task of knowing what happens in the virtual laboratory These data are particularly relevant when analyzed in context: the data retrieved represent all the users' interactions over a long period of time, regardless of the time zone of the visitor, the specific time of connection. The data provide metrics that teachers would never know or control without the help of the system. 


\section{DISCUSSION}

This section focuses on commenting the main points shown in the article, highlighting both the lights and the shadows cast by this experience, the tools developed, and the utility that the system acquires in a learning context such as the one shown. To do so, a number of subsections are set, each of which presents a question, key in the author's view, which will be answered as concisely as possible:

\section{A. Is this proposal innovative?}

It is true that in the field of serious games there are already initiatives for the interconnection with systems like MOOCs, PLEs, VLEs, Learning Analytics platforms, etc. [28-33], but in the realm of virtual worlds there are not many such initiatives [34], since a review of the current literature reveals that there is a tendency in the approach to proposals for the integration of different systems like VLEs, PLEs, architectures and applications together with Virtual Worlds [28, 35-39], but not with systems that analyze the performance of students within virtual practicals, or analyzing (however basic it may even be considered) the interaction and the evidences collected from student usage of 3D learning scenarios and practices. The literature mainly describes the interconnection of Virtual Worlds environments like OpenSim or Second Life with widespread platforms in the current teaching (Moodle, mainly) by using Sloodle and other platforms, so that these 3D environments extend their horizons of educational usage based on the support of systems and platforms where teachers already perform constant work creating materials and using them as an aid to teaching.

In fact, the authors of this paper believe that this system is a true innovation in the field of teaching in virtual worlds (and even more in the field of education in Health Sciences) because of its features and vision outside the mere connection with systems that provide learning objects or materials created by teachers, but as an aid to the evaluation and understanding of how students perform in such complex and dynamic environments like these [40]. Even in the most recent literature it is possible to find authors (Griebel et al [41], for example) that describe the proposal of this Usalpharma architecture as an interesting contribution that can open up new possibilities in the use of external systems completing the educational use of virtual worlds through the evidence collection system, the real-time responses both within the Virtual World and outside it, and its possibilities of use for the evaluation of activities. Therefore, for the authors it is possible to consider it a real, innovative and viable proposition for help in teaching. The interconnection of this architecture (using the services already available and others to be built) with other applications extends its field of education and eLearning, and could help to achieve better results in learning scenarios arranged in $3 \mathrm{D}$ environments of this type.

\section{B. Is this proposal helpful?}

The usefulness of such systems, from the point of view of the authors, depends primarily on the problem that it helps to solve. In the case of Usalpharma laboratory, this proposal provides information that cannot be achieved otherwise within a virtual world like Second Life, but it is necessary to consider whether this information is vital or not in a process of teaching and evaluation of students. In this case, the authors agree that, in the present case, the information is not vital, since evaluation depends mostly on the report submitted by the students (it represents $75 \%$ of the final mark); therefore, this system only provides extra assessment elements. What must be noted is that, in this particular case, those extra elements are also designed to establish a right to assessment or not (the reader will remember the conditions set for the assessment of the practical, of at least $12 \%$ of proven elements and a minimum time of interaction with the virtual laboratory of one hour). Moreover, the authors also wish to state that the contribution made by the system in the form of information for students and teachers is a faithful overview of what happens in a environment like a virtual world, allowing teachers to measure aspects such as dedication (hours used), effort (number of sessions, number of actions to detect all elements that must be evaluated) or persistence (number of sessions used, evolution of the completion of the activity in each session, etc.), for example. This type of data, although not taken into account in the specific assessment for this practical work (and therefore the architecture is not decisive in the present evaluation) could be considered as catalyst values for the final evaluation. The data were part of the rubric for assessing practice work, giving specific metrics to aspects that are not typically measured in a clear way. In summary, although the system has not a vital utility for the practical work, it is increasingly being consolidated as a helper to the teacher. Additionally, the system is aligned with the current trends of tools, applications and utilities that are opening new paths in new learning contexts that will be relevant in technology-enhanced learning in the future..

\section{Does it help to improve teaching?}

In this sense, it is difficult to provide a clear answer. By the data in Table 1, it is possible to say that the shift of paradigm and support in this case from classroom guidance to a system that provides feedback to students automatically, has not broken the trend of improved results, even when the results were improved significantly in the last year. The paradigm shift provided by this system is helping to improve teaching in a context such as a Virtual World, allowing students to experience completely immersive environment like Second Life and avoiding the guidance of the teacher in real time. However, venturing this conclusion would be going too far as it is possible that other factors affect results. Among the factors that could be relevant for the improvement of the results, can be highlighted the smaller number of participant students in the last years, the 
students' better predisposition towards such tools, the previous experience of some students with this kind of audit simulation, etc.

In any case, time will determine whether this system or others like this will significantly and unequivocally improve the students' learning and results, or automation of certain tasks previously performed by the teacher, are not really replaceable, and these systems simply must serve teachers as an aid in the acquisition of knowledge and decision making.

\section{Can the system have more applications in addition to} those described?

This proposal is, even today, a proof of concept on the use and application of such systems and platforms related to eLearning. The authors strongly believe that this proposal could have a more intensive usage and increased functionality through integration with other systems and eLearning platforms, besides using more advanced algorithms and analysis techniques than those currently applied, so that they could get to perform complex learning analysis, behavioral analysis (which is already done in Virtual Worlds in other ways [40]), determination of the learning path for each student, personalizing learning, development of adaptive systems within Virtual Worlds, copy-detection among practices performed in 3D environments, etc.

\section{CONCLUSIONS AND FUTURE WORK}

In this last section are presented the conclusions and future lines of work that arise within the project Usalpharma.

\section{A. Conclusions}

This article shows how a software architecture has been conceived, designed, implemented and tested that helps support an educational activity in a learning environment like a Virtual World. The article delves into the problems that such systems can solve as well as the benefits, profit, exploitation thereof, or the improvement achieved from current systems and methodologies.

From a standpoint of software engineering, this paper describes how a team of engineers has been able to develop an innovative solution to a problem of some complexity, showing what tools and methods they followed to overcome the difficulties, how they planned the development and use of the solution from a multi-platform view.

From a standpoint of eLearning, this work shows how a multidisciplinary team has been able to design, build and put in use a solution to a real and specific learning problem of graduate students from the branch of knowledge of Health Sciences and Pharmacy. In addition, from this point of view, this work shows the results that have been achieved in the first two years that the proposal has been used, and how these results have been satisfactory and provide real value to the activity of teachers and students.

\section{B. Future work}

As future work to improve and expand this architecture and its use, it is possible to distinguish three major ways:

- In the short-term the authors plan to increase the metrics shown and calculated (e.g., see how many times a student consults his or her progress, the distribution over time of the students' effort with their practicals, etc.). The authors will also continue using the solution described in this article in successive editions of the subject Quality Assurance in the Laboratory Analysis in the Pharmaceutical Industry within the Master in Drugs Evaluation and Development of the University of Salamanca and expand its use to other subjects that use this type of teaching methodologies through Virtual Worlds, to continue testing the solution in different contexts and assess more users (getting better measures on usefulness of these tools for teachers).

- In the medium term, the authors want to create a set of APIs and utilities, allowing the interconnection of this system with other platforms, so that the proposed architecture will serve as a facilitator of data and metrics that can be expanded, used or integrated into current or future eLearning systems to help close the loop between the various technological tools that help learning any subject.

In the long term, the authors plan the possibility of integrating the system with other standard RDF specifications and recommendations. Among them they propose the integration of the description of the interactions, the description of objects and users through Open Linked Data [42], following the schemes of the Linked Data Platform recommendation of the W3C [43]. Thus, the architecture and its components could eventually contain all the information concerning the activity and can have descriptions associated with each object, each target, maintaining user profiles under specific linkable URIs, etc., allowing mapping the domain of the problem and exploiting all the information from clients and developed platforms as well as from potential third-party applications connected to services that will provide the architecture.

\section{ACKNOWLEDGMENTS}

The authors wish to thank the Vice Chancellor for Academic Policy at the University of Salamanca (Spain) for funding the Teaching Innovation projects ID2013/201 and ID2014/0164 that supported the development of the software architecture and software solutions presented in this paper. Also, the author Juan Cruz-Benito would like to thank the European Social Fund and the Consejería de Educación of the Junta de Castilla y León (Spain) for funding his pre-doctoral fellow contract. 


\section{REFERENCES}

F. J. García-Peñalvo, M. Á. Conde González, and S. Bravo Martín. (2009, 10/02/2015). Ingeniería del Software: Introducción. Available: http://ocw.usal.es/ensenanzas-tecnicas/ingenieria-delsoftware/contenidos/Tema0-Sumario-1pp.pdf

[2] IEEE, "IEEE Standard Glossary of Software Engineering Terminology," in IEEE Std 610.12-1990, ed, 1990, pp. 1-84.

[3] J. Cruz-Benito, R. Therón, and F. J. García-Peñalvo, "Software Architectures Supporting Human-Computer Interaction Analysis: A Literature Review," in Learning and Collaboration Technologies, ed: Springer International Publishing Switzerland, In press.

[4] Linden Lab. (2014, Feb 28, 2014). Second Life. Available: http://secondlife.com

[5] C. Maderuelo, A. Martin-Suarez, J. S. Pérez-Blanco, H. Zazo, J. Cruz-Benito, and A. Domínguez-Gil, "Facility-based inspection training in a virtual 3D laboratory," Accreditation and Quality Assurance, vol. 19, pp. 403-409, 2014/10/01 2014.

[6] C. Maderuelo, J. S. Pérez-Blanco, H. Zazo, J. M. Armenteros del Olmo, J. Cruz Benito, and A. Martín-Suárez, "Auditing Training Practice To Postgraduate Pharmacy Students In A Virtual World," presented at the 19th Annual Conference of the European Association of Faculties of Pharmacy (EAFP 2013), Ankara, Turkey, 2013.

[7] C. Maderuelo, J. S. Pérez-Blanco, J. M. Armenteros del Olmo, H. Zazo, D. Sánchez Vicente, J. Cruz-Benito, et al., "Satisfacción de estudiantes de Postgrado con una práctica en Second Life," presented at the Congreso Virtual Mundial de e-Learning 2012, 2012

[8] L. Jarmon, K. Lim, and B. Carpenter, "Introduction: Pedagogy, education and innovation in virtual worlds," Journal of Virtual Worlds Research, vol. 2, pp. 3-4, 2009.

[9] J. Shen and L. B. Eder, "Intentions to use virtual worlds for education," Journal of Information Systems Education, vol. 20, p. 225, 2009.

[10] M. Varvello, S. Ferrari, E. Biersack, and C. Diot, "Exploring second life," IEEE/ACM Transactions on Networking (TON), vol 19, pp. 80-91, 2011.

[11] M. Virvou and G. Katsionis, "On the usability and likeability of virtual reality games for education: The case of VR-ENGAGE," Computers \& Education, vol. 50, pp. 154-178, 2008.

[12] Q. Wang, "Design and evaluation of a collaborative learning environment," Computers \& Education, vol. 53, pp. 1138-1146, $12 / / 2009$.

[13] J. Cruz, R. Therón, E. Pizarro, and F. J. García-Peñalvo, "Knowledge Discovery in Virtuah Worlds Usage Data: approaching Web Mining concepts to 3D Virtual Environments," Proceedings Fourth International Workshop on Knowledge Discovery, Knowledge Management and Decision Support (Eureka-2013), 2013.

[14] F. J. García-Peñalvo, J. Cruz-Benito, C. Maderuelo, J. S. PérezBlanco, and A. Martín-Suárez, "Usalpharma: A Cloud-Based Architecture to Support Quality Assurance Training Processes in Health Area Using Virtual Worlds," The Scientific World Journal, vol. 2014, 2014.

[15] Linden Lab. (2015, 28/07/2015). APIs and Web Services Portal. Available:

http://wiki.secondlife.com/wiki/APIs and_Web_Services_Portal

[16] Linden Lab. (2015, 15/03/2015). Second Life. Terms of Service. Available: http://www.lindenlab.com/tos

[17] Á. del Blanco Aguado, J. Torrente, I. Martínez-Ortiz, and B. Fernández-Manjón, "Análisis del Uso del Estándar SCORM para la Integración de Juegos Educativos," Revista Iberoamericana de Tecnologías del/da Aprendizaje/Aprendizagem, p. 118, 2011.

[18] A. del Blanco, A. Serrano, M. Freire, I. Martínez-Ortiz, and B. Fernández-Manjón, "E-Learning standards and learning analytics. Can data collection be improved by using standard data models?," in Global Engineering Education Conference (EDUCON), 2013 IEEE, 2013, pp. 1255-1261.

[19] World Wide Web Consortium (W3C). (2012, 15/03/2015). RDF Interfaces. A set of basic primitives and a low-level API for working with RDF data. Available: http://www.w3.org/TR/2012/NOTE-rdf-interfaces-20120705/

[20] World Wide Web Consortium (W3C). (2004, 15/03/2015). RDF Semantics. W3C Recommendation 10 February 2004. Available: http://www.w3.org/TR/2004/REC-rdf-mt-20040210/

[21] World Wide Web Consortium (W3C). (2014, 15/03/2015). HTTP - Hypertext Transfer Protocol. Available: http://www.w3.org/Protocols/

[22] MongoDB. (2015, 15/03/2015). Website. Available: https://www.mongodb.org/

[23] J. Cruz-Benito, F. J. García-Peñalvo, and R. Therón, "Defining generic data collectors for Learning Analytics: Facing up the heterogeneous data from heterogeneous environments," presented at the International Conference on Advanced Learning Technologies (ICALT) 2014, Athens, Greece. , 2014.

[24] MongoDB, "Top 5 Considerations When Evaluating NoSQL Databases," ed, 2013.

[25] Django Software Foundation. (2015, 15/03/2015). Django Web Framework. Available: https://www.djangoproject.com/

[26] J. Cruz-Benito, F. J. Garcia-Peñalvo, R. Theron, C. Maderuelo, J. S. Perez-Blanco, H. Zazo, et al., "Using software architectures to retrieve interaction information in eLearning environments," in Computers in Education (SIIE), 2014 International Symposium on, 2014, pp. 117-120.

[27] J. Cruz-Benito, R. Therón, F. J. García Peñalvo, C. Maderuelo, J. S. Pérez-Blanco, H. Zazo, et al., "Monitoring and feedback of Learning Processes in Virtual Worlds through analytics architectures: A real case," in Sistemas y Tecnologías de Información. Actas de la $9^{a}$ Conferencia Ibérica de Sistemas y Tecnologías de Información. vol. I Artículos, Á. Rocha, D. Fonseca, E. Redondo, L. P. Reis, and M. P. Cota, Eds., ed Barcelona, España, June, 18-21, 2014: AISTI (Asociación Ibérica de Sistemas y Tecnologías de Información), 2014, pp. 1126-1131.

[28] L. Stoicu-Tivadar, V. Stoicu-Tivadar, D. Berian, S. Drăgan, A. Serban, and C. Serban, "eduCRATE-A Virtual Hospital Architecture," Studies in health technology and informatics, vol. 205, pp. 803-807, 2013.

[29] Á. Serrano-Laguna, J. Torrente, P. Moreno-Ger, and B. FernándezManjón, "Application of learning analytics in educational videogames," Entertainment Computing, vol. 5, pp. 313-322, 2014.

[30] Á. Serrano-Laguna, J. Torrentea, B. Maneroa, Á. del Blancoa, B. Borro-Escribanoa, I. Martínez-Ortiza, et al., "Learning Analytics and Educational Games: Lessons Learned from Practical Experience," in Games and Learning Alliance, ed: Springer, 2014, pp. 16-28.

[31] J. Cruz-Benito, O. Borrás-Gené, F. J. García-Peñalvo, Á. Fidalgo Blanco, and R. Therón, "Detection of Non-Formal and Informal Learning in learning communities supported by social networks in the context of a Cooperative MOOC," in Proceedings of the XVII International Symposium on Computers in Education (SIIE'15) (Setúbal, Portugal. 25-27 November 2015), M. d. R. r. Rodrigues, M. n. Llamas Nistal, and M. Figueiredo, Eds., ed Portugal: IEEE, 2015.

[32] J. Cruz-Benito, O. Borrás-Gené, F. J. García-Peñalvo, Á. Fidalgo Blanco, and R. Therón, "Extending MOOC ecosystems using web services and software architectures," in Proceedings of the XVI International Conference on Human Computer Interaction, ed Vilanova i la Geltrú, Spain: ACM, 2015, pp. 1-7.

[33] F. J. García-Peñalvo, J. Cruz-Benito, O. Borrás-Gené, and Á. Fidalgo Blanco, "Evolution of the Conversation and Knowledge Acquisition in Social Networks Related to a MOOC Course," in Learning and Collaboration Technologies. vol. 9192, P. Zaphiris and A. Ioannou, Eds., ed: Springer International Publishing, 2015, pp. 470-481.

[34] J. Cruz-Benito, C. Maderuelo, F. J. García-Peñalvo, R. Therón, J. S. Pérez-Blanco, H. Zazo, et al., "Usalpharma: Una arquitectura software al servicio del aprendizaje en Mundos Virtuales," VAEP RITA, vol. 3, pp. 148-159, 2015.

[35] D. Livingstone and J. Kemp, "Integrating web-based and 3D learning environments: Second Life meets Moodle," Next Generation Technology-Enhanced Learning, vol. 8, 2008. 
[36] J. W. Kemp, D. Livingstone, and P. R. Bloomfield, "SLOODLE: Connecting VLE tools with emergent teaching practice in Second Life," British Journal of Educational Technology, vol. 40, pp. 551-555, 2009.

[37] E. P. Lucas, J. Cruz-Benito, and O. G. Gonzalo, "USALSIM: learning, professional practices and employability in a 3D virtual world," International Journal of Technology Enhanced Learning, vol. 5, pp. 307-321, 2013.

[38] A. Konstantinidis, T. Tsiatsos, S. Demetriadis, and A. Pomportsis, "Collaborative learning in OpenSim by utilizing sloodle," in Telecommunications (AICT), 2010 Sixth Advanced International Conference on, 2010, pp. 90-95.

[39] P. Kallonis and D. G. Sampson, "A 3D Virtual Classroom Simulation for Supporting School Teachers Training Based on Synectics-" Making the Strange Familiar"," in Advanced Learning Technologies (ICALT), 2011 11th IEEE International Conference on, 2011, pp. 4-6.

[40] J. Cruz-Benito, R. Therón, F. J. García-Peñalvo, and E. Pizarro Lucas, "Discovering usage behaviors and engagement in an Educational Virtual World," Computers in Human Behavior, vol. 47, pp. 18-25, 6// 2015.

[41] L. Griebel, H.-U. Prokosch, F. Köpcke, D. Toddenroth, J. Christoph, I. Leb, et al., "A scoping review of cloud computing in healthcare," BMC medical informatics and decision making, vol. 15, p. 17, 2015.

[42] C. Bizer, T. Heath, and T. Berners-Lee, "Linked Data - The Story So Far," Int. J. Semantic Web Inf. Syst., vol. 5, pp. 1-22, / 2009.

[43] World Wide Web Consortium (W3C). (2015, 15/03/2015). Linked Data Platform 1.0. W3C Recommendation 26 February 2015. Available: http://www.w3.org/TR/2015/REC-ldp-20150226/

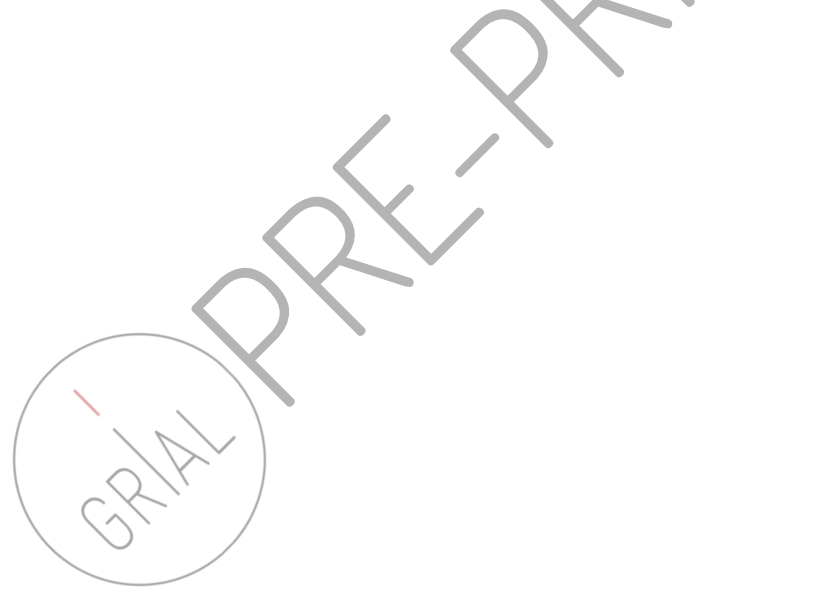




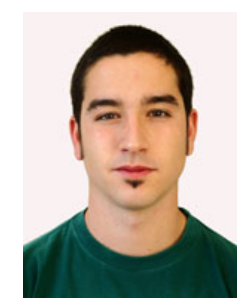

Juan Cruz-Benito received the M.Sc. degree in intelligent systems from the University of Salamanca, Spain, in 2013, where he is currently pursuing the $\mathrm{Ph} . \mathrm{D}$. degree in computer sciences. He is one of the youngest members of the Research Group Interaction and eLearning, where he specializes in software solutions based on technology ecosystems. He is an expert in educational vir-tual worlds, data analytics, and technologies for educational purposes, and disciplines that he has developed in many innovation and research projects. He has participated in many European and national projects, such as Tagging, Recognition and Acknowledgment of Informal Learning Experiences, Virtual Alliances for Learning Society, USALSIM Virtual Campus, or the Spanish Observatory for University Employability and Employment, where he participated as a Software Engineer, Researcher, and Developer.

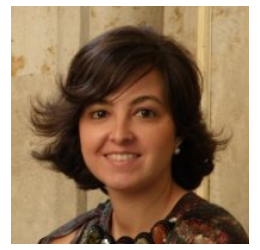

Cristina Maderuelo received the degree in Pharmacy from the University of Salamanca, specializing in Industrial Pharmacy and Pharmaceutical Technology at the University of Barcelona, and $\mathrm{PhD}$ from the University of Salamanca. Combines her work as Galenic Technical Developer within the Department of Pharmacy and Pharmaceutical Technology of the University of Salamanca with her teaching activity as Associate Professor at the same university. Cristina is also one of the main promoters of the group Teaching Innovation USALPHARMA, where she directs the teaching activities carried out in the virtual laboratory Usalpharma Lab. She has participated in many projects of pharmaceutical R \& D and projects Educational Innovation, publishing the results of both lines of research in many congresses and international magazines.

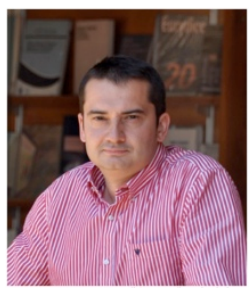

Francisco José García-Peñalvo received the degrees in computing from the University of Salamanca and the University of Valladolid, and the Ph.D. degree from the University of Salamanca. He is currently the Head of the Research Group Interaction and eLearning. He has led and participated in over 50 research and innovation projects. He was the Vice Chancellor of Innovation with the University of Salamanca from 2007 to 2009. He has authored over 300 articles in international journals and conferences. His main research interests focus on eLearning, computers and education, adaptive systems, Web engineering, semantic Web, and software reuse. He was a Guest Editor of several special issues of international journals, such as Online Information Review, Computers in Human Behavior, and Interactive Learning Environments. He is also a member of the Program Committee of several international conferences and a Reviewer of several international journals. He is currently the Editor-in- Chief of the International Journal of Information Technology Research and the Education in the Knowledge Society Journal. Besides, he is the Coordinator of the multidisciplinary Ph.D. Programme on Education in the Knowledge Society.

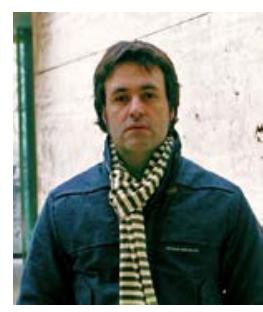

Roberto Therón studied Informatics at the University of Salamanca (Diploma) and the University of La Coruña (BA). After joining the Research Group Robotics at the University of Salamanca, he presented his thesis work, "Parallel calculation configuration space for redundant robots", receiving the Extraordinary Doctoral Award. He subsequently obtained the degrees of Bachelor in Communication Studies (University of Salamanca) and Bachelor in Humanities (University of Salamanca). At the same University of Salamanca he continues to carry out his research work, as manager of VisUsal group (within the Recognized Research Group MIDA) which focuses on the combination of approaches from computer science, statistics, Graphic Design and Information Visualization for obtain an adequate understanding of complex data sets. In recent years, he has been dedicated to developing advanced visualization tools for multidimensional data, such as genetic or paleo-climate data. In the field of Visual Analytics he develops productive collaborations with groups and institutions internationally recognized as the Laboratory of Climate Sciences and the Environment (Paris) or the Center for Advanced Visual Analytics UN
(Switzerland). He is the author of over 70 articles in international journals and conferences.

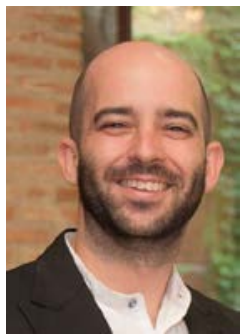

Jonás Samuel Pérez-Blanco holds a degree in Pharmacy and a MSc in Design, Procurement and Drug Evaluation at the University of Salamanca; he currently is an assistant professor of Pharmacy and Pharmaceutical Technology of the University of Salamanca where he is doing a doctoral thesis on monitoring of anticancer drugs. His concentrated trajectory focuses on optimizing pharmacotherapeutic treatments and teaching innovation in the pharmaceutical field. Samuel is the coordinator of new technologies at the Faculty of Pharmacy; He has directed and collaborated on more than 20 research projects and national and international educational innovation such as COPHELA project (Cooperation in Quality Assurance Pharmacy Education and Training between Europe and Latin America). He has also been honored with several awards for his participation in projects of both biomedical character (Academy of Medicine of Valladolid, Spain) and teaching innovation (Academy of Pharmacy of Castilla y Leon, Spain) and has presented the results of his research in journals high impact international as well as national and international conferences.

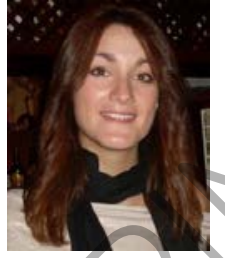

Hinojal Zazo Gómez is a graduate in Pharmacy and Masters in Design, Preparation and Drugs Evaluation at the University of Salamanca. She is currently a Ph.D. student in pharmacy, within the same university, funded by a grant from the Ministry of Education of the Spanish Government. She simultaneously participates in the teaching innovation group Usalpharma. In 2012 she received the Award of the Academy of Pharmacy of Castile and Leon for her work subsequently published in the International Jounal of Antimibrobial Agents. Hinojal has experience in diverse fields such as HPLC analysis, cell cultures or teaching through Virtual Worlds by participating in multiple projects for Research and Innovation, and the subject taught in subjects of the degree of Pharmacy and various courses offered by the University of Salamanca in relation to this area.

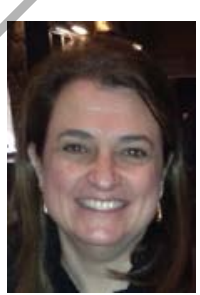

Ana Martín Suárez has a PhD in Pharmacy and is a Professor in the Department of Pharmacy and Pharmaceutical Technology at the University of Salamanca. She completed her training as a researcher in the Department of Pharmacy and Pharmaceutical Technology and service of Pharmacy of the University Hospital of Salamanca. She belongs to the GIR of Experimental and Clinical Pharmacokinetics at the University of Salamanca and IBSAL (Institute of Biomedicine at the University of Salamanca). Her main research is in the field of Clinical Pharmacokinetics, addressing drug monitoring in biological fluids and population pharmacokinetic analysis, modeling and simulation PK / PD (Pharmacokinetic / Pharmacodynamic). She has published more than 30 articles, most included in the Journal Citation Reports. With the group of teaching innovation Usalpharma she has developed numerous projects, published articles, conducted training of teachers and received several awards. 